\title{
Ernährungsempfehlungen bei Diabetes mellitus (Update 2019)
}

\author{
Karin Schindler · Johanna Brix · Sabine Dämon · Friedrich Hoppichler · Alexandra Kautzky-Willer • \\ Renate Kruschitz · Hermann Toplak · Bernhard Ludvik
}

Zusammenfassung Es besteht breiter Konsens, dass eine effiziente Diabetes-Therapie in den meisten Fällen auch von einer Modifikation des Lebensstils begleitet sein muss. Das bedeutet für die Patienten in der Regel eine Normalisierung des Körpergewichts (Gewichtsreduktion und/oder Halten des Gewichts) in Kombination mit einer Veränderung des Bewegungsund Ernährungsverhaltens.

Die medizinische Ernährungstherapie ist integraler Teil des Diabetesmanagements und soll individualisiert und PatientInnen-zentriert erfolgen. Das Ziel ist die positive Beeinflussung des postprandialen Glukoseanstiegs sowie insgesamt des Glukose- und

\section{K. Schindler $(\bowtie) \cdot$ A. Kautzky-Willer}

Klinische Abteilung für Endokrinologie und Stoffwechsel, Universitätsklinik für Innere Medizin III, Medizinische Universität Wien, Währinger Gürtel 18-20, 1090 Wien, Österreich

karin.schindler@meduniwien.ac.at

\section{J. Brix · B. Ludvik}

1. Medizinische Abteilung mit Diabetologie, Endokrinologie und Nephrologie, Krankenanstalt Rudolfstiftung, Wien, Österreich

\section{F. Hoppichler}

Abteilung für Innere Medizin, Krankenhaus der Barmherzigen Brüder Salzburg, Salzburg, Österreich

\section{H. Toplak}

Klinische Abteilung für Endokrinologie und Diabetologie,

Universitätsklinik für Innere Medizin, Medizinische

Universität Graz, Graz, Österreich

\section{S. Dämon · F. Hoppichler}

Special Institute for Preventive Cardiology and Nutrition, SIPCAN - Initiative für ein gesundes Leben, Salzburg, Österreich

\section{R. Kruschitz}

Abteilung für Innere Medizin, Krankenhaus der

Elisabethinen, Klagenfurt, Österreich
Fettstoffwechsels, die Gewichtskontrolle sowie die Prävention bzw. Verzögerung von T2DM assoziierten Komplikationen.

Schlüsselwörter Körpergewicht • Kohlenhydratzufuhr · Proteinzufuhr · Fettzufuhr · Mikronährstoffe

\section{Nutrition for diabetic patients (Update 2019)}

Summary Evidence demonstrates that medical diabetes treatment has to be accompanied by lifestyle modifications. Structured nutrition interventions and increased physical activity will help patients to normalise, respectively maintain their body weight.

The main target of a medical nutrition therapy aims at achieving normal or nearly normal blood glucose levels, prevention of delay of diabetes associated complications.

Keywords Body weight $\cdot$ Dietary carbohydrates $\cdot$ Dietary protein $\cdot$ Dietary fats $\cdot$ Micronutrients

$\begin{array}{ll}\text { Abkürzungen } \\ \text { ADI } & \text { „Acceptable daily intake“ } \\ \text { ALA } & \text { Alpha-Linolensäure } \\ \text { BMI } & \text { Body Mass Index } \\ \text { GI } & \text { Glykämischer Index } \\ \text { GL } & \text { Glykämische Last } \\ \text { HDL } & \text { High density Lipoprotein } \\ \text { KHK } & \text { Koronare Herzerkrankung } \\ \text { LCHF } & \text { low carb, high fat } \\ \text { LDL } & \text { Low density Lipoprotein } \\ \text { PUFA } & \text { Mehrfach ungesättigte Fettsäuren } \\ \text { T2DM } & \text { Diabetes mellitus Typ 2 }\end{array}$

Abkürzungen

Glykämische Last

KHK

LCHF low carb, high fat

PUFA Mehrfach ungesättigte Fettsäuren

T2DM Diabetes mellitus Typ 2 


\section{Grundsatzstatement}

Es besteht breiter Konsens, dass übergewichtige und adipöse Patienten mit Diabetes mellitus ihr Gewicht normalisieren sollten [1]. Um dies zu erreichen und das Gewicht auch zu halten, ist in der Regel eine Modifikation des Lebensstils, einschließlich des Ernährungs- und Bewegungsverhalten nötig [2]. Die positive Beeinflussung des Glukose- und Fettstoffwechsels, die Normalisierung des Körpergewichts und die Prävention bzw. Verzögerung von T2DM-assoziierten Komplikationen kann durch folgende ernährungstherapeutische Maßnahmen erreicht werden:

- Gemüse, Hülsenfrüchte, Vollkornprodukten als grundlegende Kohlenhydratquellen.

- Eine Reduktion der Aufnahme von Mono- und Disacchariden (z.B. zuckerreiche Getränke, Süßigkeiten und Mehlspeisen) erleichtert das Erreichen einer ausgeglichenen bzw. negativen Energiebilanz und damit die Gewichtstabilisierung bzw. eine Gewichtsreduktion.

- Eine Reduktion der Fettzufuhr kann indiziert sein. Für die Auswirkung der Nahrungsfette auf den Stoffwechsel ist jedoch nicht nur die verzehrte Menge von Bedeutung, sondern die Fettqualität. Pflanzliche Öle, reich an einfach und mehrfach ungesättigten Fettsäuren, sind den tierischen Fetten vorzuziehen.

- Für die Empfehlung einer dauerhaft erhöhten Proteinzufuhr zur Gewichtsnormalisierung gibt es derzeit noch unzureichende Evidenz.

- Die ausreichende Aufnahme von Mikronährstoffen (Vitaminen, Mineralstoffen und Spurenelementen) ist ein wichtiger Faktor zur Erhaltung der Gesundheit von Typ 1 und Typ 2 Diabetikern. Die empfohlene tägliche Zufuhr unterscheidet sich nicht von jener für gesunde Erwachsene. Nährstoffdichte Lebensmittel (i. e. reich an Vitaminen, Mineralstoffen und Spurenelementen) sollten daher bevorzugt werden.

- Der Konsum von alkoholischen Getränken sollte moderat sein (Frauen: maximal ein, Männer maximal zwei Getränke/Tag).

Fest steht, es gibt keine „One-Size-Fits-All“ Lösungen. Eine Ernährungstherapie muss individualisiert und vorurteilsfrei sein und vermittelt lebensmittelbasierte Empfehlungen [3, 4]. Studien, die gesamte Ernährungsmuster betrachten, geben Hinweise, dass eine mediterrane Ernährungsweise bzw. andere Ernährungsmuster, die ebenfalls reich an Gemüse, zuckerarmen Obst und Vollkornprodukten sind, einschließlich der Verwendung von Oliven- und/oder Rapsöl, sich günstig auf die glykämische Kontrolle und das kardiovaskuläre Risiko auswirken [5-9]. Den Patienten wird die Bedeutung der Portionsgrößen erklärt, ihre persönlichen Vorlieben und Bedürfnisse, kulturelle und religiöse Aspekte, sowie ökonomische Möglichkeiten werden in der Mahlzeitenplanung be- rücksichtigt. Studien zeigen, dass eine Beratung durch Ernährungsfachkräfte mit einer effektiveren Senkung des HbAlc assoziiert ist [3]. Digitale und qualitätsgesicherte Programmangebote haben ebenfalls das Potential bei einer Ernährungsumstellung zu unterstützen [10].

\section{Nährstoffaufnahme im Detail}

\section{Kohlenhydrate und Ballaststoffe}

Typ 1 und Typ 2 Diabetiker können zwischen 45 und $60 \%$ der aufgenommenen Gesamtenergie in Form von Kohlenhydraten aufnehmen. Kohortenstudien zeigen, dass Gemüse, Vollkornprodukte, Hülsenfrüchte und Obst bevorzugt werden sollten. Der häufig beobachtete ungünstige Effekt einer stärkereichen Ernährung auf die Triglyzerid-Plasmaspiegel [11] kann vermieden werden, wenn die verzehrten kohlenhydratreichen Lebensmittel gleichzeitig ballaststoffreich sind. Daher sind Vollkorngetreideprodukte Weißmehlprodukten vorzuziehen. Eine tägliche Ballaststoffaufnahme von mindestens 25-29 g/d wird empfohlen. Höhere Aufnahmen waren mit größeren positiven Effekten assoziiert [12]. Die Hälfte der Ballaststoffe sollte in Form von löslichen Ballaststoffen aufgenommen werden (z. B. Pektine, Inulin). Diese finden sich vor allem in Gemüse und Obst. Der Verzehr von Ballaststoffen in Form von natürlichen Lebensmitteln ist dem von ballaststoffreichen Nahrungsergänzungsmitteln vorzuziehen.

Bei der Auswahl von kohlenhydratreichen Lebensmitteln ist neben dem Ballaststoffgehalt auch der Glykämische Index bzw. die Glykämische Last zu beachten. Der Einfluss von Nahrungskohlenhydraten auf die glykämische Antwort hängt von verschiedenen Faktoren wie aufgenommener Menge, Art und zellulärer Struktur, thermischer und/oder mechanischer Verarbeitung sowie gleichzeitigem Verzehr anderer Makronährstoffe ab [13]. Darüber hinaus wird die glykämische Antwort auf Nahrungsmittel auch von der Nüchternblutglukosekonzentration und dem Ausmaß der Insulinresistenz beeinflusst.

Glykämischer Index (Gl), glykämische Last (GL)

Der GI ist eine Maßzahl für die Wirksamkeit verschiedener Lebensmittel auf die Blutglukose. Seine Bestimmung erfolgt, indem die Blutzuckerkurve nach Aufnahme von $50 \mathrm{~g}$ Kohlenhydraten aus einem Testlebensmittel über $2 \mathrm{~h}$ verfolgt wird. Diese Kurve wird $\mathrm{zu}$ jener, die sich aus dem Konsum von $50 \mathrm{~g}$ Kohlenhydraten in Form von Weißbrot oder Glukose ergibt, in Beziehung gesetzt. Der GI wird in Prozent in Bezug zum Referenzlebensmittel angegeben. Daher bedeutet ein $\mathrm{GI}=70$, dass die Blutzuckerwirksamkeit des untersuchten Lebensmittels $70 \%$ der von $50 \mathrm{~g}$ Weißbrot bzw. Glukose beträgt (die Fläche unter der Blutzuckerkurve ist um 30\% kleiner als die von Weißbrot bzw. Glukose). Die Auswirkungen eines Lebens- 
mittels auf den Blutglukose- und Insulinspiegel hängen sowohl von der Menge der verzehrten Kohlenhydrate als auch vom GI ab. Die alleinige Betrachtung des GI hat den Nachteil, dass er sich definitionsgemäß auf $50 \mathrm{~g}$ Kohlenhydrate bezieht, was nicht immer die realen Verzehrgewohnheiten wiederspiegelt. So entsprechen $50 \mathrm{~g}$ Kohlenhydrate aus Karotten einer Menge von $650 \mathrm{~g}$, so dass der Verzehr einer üblichen Portion zwischen 100-150g trotz des höheren GI geringe Auswirkungen auf den Blutglukose-Spiegel hat. Die Verzehrgewohnheiten werden im Konzept der GL berücksichtigt. Die GL errechnet sich aus dem Produkt der verwertbaren Kohlenhydratmenge pro Portion und dem GI [14].

Neben dem Einfluss des GI auf die metabolische Kontrolle wird auch eine generelle Reduktion der Kohlenhydrataufnahme zur Verbesserung der Stoffwechsellage diskutiert. Diese Reduktion wird üblicherweise dann unter dem Terminus „Low Carb Diät“ subsummiert. Der Ausdruck „low carb“ ist eigentlich falsch es müsste „low carb high fat“ (LCHF) Diät genannt werden [15]. Nach derzeitigem Wissenstand spricht man von einer LCHF Diät, wenn 50-150 g Kohlenhydrate pro Tag verzehrt werden. Eine ketogene Diät, die Extremform der LCHF Diät, erlaubt einen Kohlenhydratverzehr von 20-50g pro Tag [16, 17]. Ziel der LCHF-Ernährung bzw. ihrer Extremform, der ketogenen Ernährung, ist, dass durch die Kohlenhydratreduktion weniger Glukose als Energielieferant zur Verfügung steht, der Insulinspiegel sinkt und der Körper durch Lipolyse Energie gewinnt, dies führt auf längere Sicht zu Exsikkose. Nach dieser Hypothese müsste es einen Wert geben, ab dem diese metabolischen Veränderungen auftreten. Eine Kohlenhydratreduktion auf unter $45 \%$ der aufgenommenen Energie kann zu Therapiebeginn mit einer stärkeren Reduktion des HbAlc assoziiert sein. Langfristig ist sie einer Diät mit einem höheren Kohlenhydratanteil nicht überlegen [13, 18]. Zum jetzigen Zeitpunkt fehlen gute Vergleichsstudien, ob eine LCHF-Ernährung einer „low fat, high carb“Ernährung bei Patienten mit Diabetes wirklich zu bevorzugen ist. Eine Metanalyse zeigte, dass zumindest über einen kurzen Zeitraum eine LCHF-Diät zu einer Verbesserung der Blutzuckereinstellung und zu einer Gewichtsabnahme bei Patienten mit T2DM führt [19]. Noch deutlicher sind diese Resultate bei einer ketogenen Diät sichtbar. Allerdings, wie bei allen extremen Ernährungsformen, ist die Therapieadhärenz eingeschränkt. Darüber hinaus ist zu bedenken, dass die meisten PatientInnen eine Kohlenhydratreduktion durch eine höhere Fettaufnahme kompensieren. Bei Nichtbeachtung der Qualität der Kohlenhydrate und Fette könnte das langfristige Risiko für kardiovaskuläre Erkrankungen steigen.

Für alle Patienten mit einem Insulinmangeldiabetes, insbesondere einem Typ 1 Diabetes, ist eine ketogene Diät nicht zu empfehlen, da das Risiko einer Ketoazidose aufgrund einer zu drastischen Insulinreduktion nicht unterschätzt werden darf. Dies kann besonders gefährlich sein, wenn diese Patienten mit SGLT2-Hemmern (bei Typ 1 Diabetes wäre dies allerdings „Off-Label“) behandelt werden.

Gesamt gesehen dürfte also immer noch die Gesamtkalorienaufnahme der beste Prädiktor für $\mathrm{Ge}$ wichtsverlust und Verbesserung der glykämischen Stoffwechsellage sein und nicht eine alleinige Reduktion der Kohlenhydrate [15].

\section{Zucker}

Eine vollständige Saccharoserestriktion wird heute nicht mehr gefordert. Zucker kann bei befriedigender Blutglukoseeinstellung in Form von Mono- und Disacchariden (max. $50 \mathrm{~g} / \mathrm{d}$ ) aufgenommen werden. Die Zuckeraufnahme sollte jedoch - wie auch für gesunde Erwachsene empfohlen - 10\% der Gesamtenergie nicht überschreiten. Eine Reduktion der Aufnahme von Mono- und Disacchariden in verarbeiteten Lebensmitteln und Getränken, erleichtert das Erreichen einer ausgeglichenen bzw. negativen Energiebilanz und damit die Gewichtstabilisierung bzw. eine Gewichtsreduktion.

Eine Diät mit einem hohen Anteil an Haushaltszucker ( $>20 \%$ der Gesamttagesenergie) führt sowohl bei Nicht-Diabetikern als auch bei Personen mit Metabolischem Syndrom zu erhöhten Plasma-Triglyzeriden [20]. Die Reaktion der Triglyzeride auf Nahrungszucker ist abhängig von der aufgenommenen Menge und dem gleichzeitigen Konsum anderer Lebensmittel. Dem Zuckerkonsum von Patienten mit einem Metabolischen Syndrom (hohe Plasma-Triglyzerid-, niedrige HDL-Cholesterinspiegel) muss besondere Aufmerksamkeit gewidmet werden.

\section{Süßstoffe}

Süßstoffe können das Erreichen einer negativen Energiebilanz unterstützen [21]. Sie sind nach derzeitigem Wissen, wenn der „Acceptable Daily Intake“ (ADI) nicht überschritten wird, unbedenklich. Ein möglicher negativer Einfluss auf das Mikrobiom und die Glukosetoleranz wird diskutiert [22]. Für eine endgültige Aussage bedarf es jedoch weiterer Forschung. Es ist jedenfalls darauf $\mathrm{zu}$ achten, dass die kalorische Einsparung durch die Verwendung von nichtkalorischen Süßstoffen und Süßungsmitteln nicht über andere Lebensmittel oder Getränke kompensiert wird.

\section{Nahrungsfette und Fettsäuren}

Der Anteil der täglich aufgenommenen Energie aus Fetten sollte 35\% der Gesamtenergie nicht überschreiten. Darüber hinaus ist es von besonderer Bedeutung, die Qualität des aufgenommenen Fettes zu beachten bzw. zu modifizieren.

Maximal $10 \%$ der täglichen Gesamtenergiezufuhr dürfen, wie bei gesunden Erwachsenen, in Form von gesättigten Fettsäuren und Transfettsäuren aufgenommen werden. Transfettsäuren entstehen bei der 
Hydrogenierung pflanzlicher Öle bzw. im Pansen von Wiederkäuern. Gesättigte Fettsäuren sind vor allem in tierischen Lebensmitteln und streichfähigen Fetten zu finden. Sie sind der diätetische Faktor mit den größtmöglichen Auswirkungen auf den Serum-Cholesterinspiegel.

Die Aufnahme mehrfach ungesättigter Fettsäuren (PUFA) sollte ebenfalls 10\% der täglichen Gesamtenergieaufnahme nicht überschreiten.

Es gibt Hinweise, dass der Austausch von gesättigten Fettsäuren durch einfach- oder mehrfach ungesättigte Fettsäuren einen protektiven Effekt in der Prävention der koronaren Herzkrankheit (KHK) hat. Der Austausch der gesättigten Fettsäuren mit Kohlenhydraten senkt das Risiko hingegen nicht. Eine Modifikation der Lebensmittelauswahl und der Ernährungsgewohnheiten kann auch eine deutliche Verbesserung im Sinne der sekundären Prävention der KHK bewirken [23].

Fischöl-Supplemente können bei Patienten mit T2DM die Triglyzerid-Spiegel senken [24]. Eine generelle Supplementierung mit Fischölen bei Patienten mit Diabetes ohne ein kardiovaskuläres Risiko kann aber derzeit nicht empfohlen werden [25].

Eine tägliche Aufnahme von $5 \mathrm{~g}$ und mehr Transfettsäuren erhöht das kardiovaskuläre Risiko um 25\% [26]. In verschiedenen Studien wurde ein LDL-Cholesterin steigernder und HDL-Cholesterin senkender Effekt beobachtet. Die Frage, ob ein höherer Konsum von Transfettsäuren mit einem höheren Diabetesrisiko verbunden ist, kann derzeit nicht endgültig beantwortet werden. Die Minimierung der Aufnahme von Transfettsäuren erscheint jedenfalls angezeigt. In Europa ist ihr quantitativer Anteil in Margarinen aufgrund verbesserter Produktionsbedingungen jedoch vernachlässigbar. Zu berücksichtigen sind jedoch andere mögliche Quellen für Transfettsäuren wie Fastfood-Produkte und fettreiche Backwaren.

\section{Cholesterin}

Die Cholesterinaufnahme sollte auf $300 \mathrm{mg} / \mathrm{d}$ beschränkt werden. Bei erhöhtem LDL-Cholesterin kann die weitere Einschränkung der Aufnahme von Nahrungscholesterin sinnvoll sein. Allerdings werden interindividuell erhebliche Unterschiede hinsichtlich des Zusammenhangs zwischen Cholesterinaufnahme und -serumspiegeln beobachtet, weshalb auch die Reaktion auf eine Reduktion der Cholesterinzufuhr sehr unterschiedlich ist [27].

Die gleichzeitige Reduktion der Aufnahme von Nahrungsfett, gesättigten Fettsäuren und Cholesterin resultiert in einer sehr deutlichen Abnahme des LDL-Cholesterins, obgleich auch eine geringfügige Abnahme des HDL-Cholesterins beobachtet wird. Jedoch überwiegt der positive Effekt der Senkung des LDL-Cholesterins. Eine fettreduzierte Kost, die zusätzlich reich an pflanzlichen Lebensmitteln ist, kann Gesamt- und LDL-Cholesterin deutlicher senken als eine lediglich fettreduzierte Diät [28, 29].

\section{Protein}

Der Anteil der täglichen Proteinaufnahme an der Gesamtenergieaufnahme kann bei Patienten ohne Anzeichen einer Nephropathie 10-20\% betragen. Die durchschnittliche Proteinaufnahme der österreichischen Bevölkerung liegt deutlich über der empfohlenen Zufuhr (1-2g/kgKG/d, Ernährungsbericht vs. empfohlen $0,8 \mathrm{~g} / \mathrm{kgKG} \mathrm{D}-\mathrm{A}-\mathrm{CH}$ Empfehlungen), weshalb man auch bei Diabetikern von einer ausreichenden Versorgung ausgehen kann. Lediglich während einer energiereduzierten Diät zur Gewichtsabnahme ist darauf zu achten, dass die adäquate Proteinaufnahme sichergestellt ist.

Inwiefern eine höhere Proteinaufnahme $(>20 \%$ der täglichen Energieaufnahme) sich langfristig auf die Entwicklung einer Nephropathie auswirkt, ist noch nicht endgültig geklärt. Die Proteinaufnahme in den üblichen Mengen $(\approx 1 \mathrm{~g} / \mathrm{kgKG})$ erscheint sicher [30, 31]. Eine Beschränkung der Proteinaufnahme verzögerte die Entwicklung der Albuminurie und die Abnahme der glomerulären Filtrationsrate. Bei Typ 1 Diabetikern mit diabetischer Nephropathie ist eine geringere Proteinaufnahme mit einer verringerten $\mathrm{Al}$ buminurie und einer Abnahme der glomerulären Filtrationsrate verbunden. Der Blutglukosespiegel wird durch die Proteinaufnahme nicht erhöht, allerdings stimuliert Nahrungsprotein die Insulinsekretion [32].

In den letzten Jahren wurde der Einfluss einer proteinreichen, kohlenhydratarmen Diät auf das Ausmaß der Gewichtsabnahme sehr kontroversiell diskutiert. Energiereduzierte, proteinreiche vs. kohlenhydratreiche Diäten über sechs Monate resultierten bei Gesunden in einer signifikant besseren Gewichtsabnahme [33]. Eine randomisierte Langzeitstudie untersuchte den Einfluss einer proteinreichen Ernährung (30 E\% Protein) versus eine kohlenhydratreiche Ernährung (15 E\% Protein) auf das Körpergewicht von Probanden mit T2DM. Die proteinreiche Ernährung hatte keinen signifikant besseren Einfluss auf das Körpergewicht und den Bauchumfang [30]. Es ist anzumerken, dass eine Proteinanteil von $30 \%$ an der Gesamtenergieaufnahme nicht praktikabel zu sein scheint - im Mittel lag die Proteinaufnahme zwischen 20 und 21E\% [30]. Proteinreiche Diäten favorisieren jedoch in der Regel eine hohe Aufnahme von Cholesterin und gesättigten Fettsäuren, der Obst- und Gemüsekonsum wird stark eingeschränkt, sie müssen daher im Hinblick auf die Prävention einer Arteriosklerose kritisch betrachtet werden.

\section{Mikronährstoffe und pflanzliche Nahrungsergän- zungen}

\section{Vitamine}

Die ausreichende Aufnahme von Mikronährstoffen (Vitaminen und Spurenelementen) ist ein wichtiger Faktor zur Erhaltung der Gesundheit von Typ 1 
und Typ 2 Diabetikern. Die empfohlene tägliche Zufuhr unterscheidet sich nicht von der für gesunde Erwachsene. Lebensmittel, die reich an Vitaminen und Spurenelementen sind, sollten daher bevorzugt werden.

Eine Langzeit-Metformingabe kann mit einem erniedrigten Vitamin-B12-Spiegel assoziiert sein. Die Einnahme von Protonenpumpenhemmern kann die Bioverfügbarkeit von Vitamin B12 darüber hinaus reduzieren. Eine regelmäßige laborchemische Kontrolle und bei Bedarf eine Supplementierung von Vitamin B12 kann sinnvoll sein.

Da Diabetes mit erhöhtem oxidativem Stress verbunden ist, erscheint es möglich, dass bei schlecht kontrolliertem diabetischem Stoffwechsel der Bedarf an Antioxidantien erhöht ist. In verschiedenen Studien wurde eine inverse Beziehung zwischen der Antioxidantienzufuhr und dem KHK-Risiko gefunden [34, 35]. Die deutlichste Beziehung bestand für Tocopherole und $\beta$-Karotin, der Effekt der Ascorbinsäure war weniger ausgeprägt. Klinische Studien, die den Effekt einer Tocopherol-Supplementierung in der Sekundärprävention der KHK untersuchten, kamen zu widersprüchlichen Ergebnissen. Die Supplementierung mit $\beta$-Karotin zeigte keinen positiven Effekt, bei Rauchern wurde sogar ein höheres Krebsrisiko gefunden. Eine Supplementierung mit Antioxidantien kann derzeit aufgrund ungeklärter Effektivität und unbekannten Langzeitfolgen nicht empfohlen werden [35].

Vor allem Schwangeren, Stillenden, älteren Patienten und solchen, die eine energiereduzierte Diät einhalten, kann eine Supplementierung mit einem Multivitaminpräparat empfohlen werden. Eine ständige Supplementierung von Mikronährstoffen in Dosierungen über der empfohlenen Tagesmaximaldosis ist besonders beim Fehlen von klinischen bzw. laborchemischen Mangelzuständen abzulehnen.

\section{Spuren- und Mengenelemente}

\section{Zink}

Zink ist als Co-Faktor der Superoxiddismutase im Radikalstoffwechsel von Bedeutung. Eine Supplementierung kann Störungen der Wundheilung positiv beeinflussen [36]. Die Evidenz für eine Supplementierung bei Diabetikern ist als unzureichend anzusehen [37].

\section{Chrom}

Eine nicht ausreichende Chromzufuhr wird mit einer gestörten Glukosetoleranz in Verbindung gebracht. Zwei randomisierte, placebokontrollierte Studien zeigten einen günstigen Effekt auf den Blutzucker. In einer rezenten Studie wurde HbAlc nicht durch eine Supplementierung mit Chrom-Picolinat verbessert [38]. Die Evidenz für eine Supplementierung ist als unzureichend anzusehen.

\section{Selen}

Der Selenstatus wird im Zusammenhang mit einem Diabetesrisiko und einem möglichen positiven Einfluss auf die glykämische Kontrolle von Diabetikern diskutiert. Sowohl ein niedriger als auch ein hoher Selen-Plasmaspiegel scheinen sich ungünstig auszuwirken (U-förmiger Zusammenhang). In der Metaanalyse von Rayman wird festgehalten, dass die Evidenz für eine routinemäßige Supplementierung von Selen nur unzureichend ist [39].

\section{Kalzium und Magnesium}

Bei älteren Patienten mit T2DM, vor allem mit niedrigem BMI, wurde eine höhere Inzidenz für Schenkelhalsfrakturen gefunden [40]. Eine optimale Kalziumresorption ist nur bei gleichzeitig verfügbarem Vitamin D erreichbar. Es gibt Hinweise, dass eine Supplementierung mit Kalzium und Vitamin D mit einem geringeren Risiko eines T2DM verbunden ist. Allerdings muss die Evidenz dafür noch als unzureichend angesehen werden.

Ein unzureichender Magnesiumstatus wird mit einer schlechten glykämischen Kontrolle bei gestörter Glukosetoleranz, [41, 42] und mit T2DM und assoziierten Komorbiditäten [43, 44] in Zusammenhang gebracht. Die ausreichende Magnesiumaufnahme scheint die Progression einer eingeschränkten Glukosetoleranz zu T2DM zu verzögern $[45,46]$. Die Magnesiumaufnahme im obersten Quartil ( $\geq 195,6 \mathrm{mg} / \mathrm{d}$ ) mit einem günstigen Effekt auf das Diabetesrisiko der Studie von Hata et al. [45] liegt deutlich unter der von den D-A-CH Fachgesellschaften empfohlenen Magnesiumaufnahme mit $300 \mathrm{mg} / \mathrm{d}$ bzw. $350 \mathrm{mg} / \mathrm{d}$ für Männer [47]. Die Evidenz für eine Magnesiumsupplementierung bei Typ 2 Diabetikern ist unzureichend.

\section{Pflanzliche Nahrungsergänzungen}

Die Verwendung von komplementären und alternativmedizinischen Produkten wird von vielen Patienten gewünscht. Zimt ist eine der von Diabetikern am häufigsten verwendeten pflanzliche Nahrungsergänzungen [48]. Zimt werden antioxidative, antiinflammatorische und antibakterielle Eigenschaften zugeschrieben. Bisher sind mehr als 200 Zimtarten bekannt [49]. Diese unterscheiden sich in der Zusammensetzung ihrer Inhaltsstoffe zum Teil signifikant. Dies und die Abhängigkeit des Gehalts der Inhaltstoffe von Klima, Wetter, Bodenbeschaffenheit und Variationen in der Herstellung machen eine Standardisierung (d.h. immer gleicher Gehalt der Wirksubstanz) von Zimt schwierig. Die Verwendung größerer Mengen von Zimt, der zum Würzen beim Kochen und Backen verwendet wird, kann daher nicht zielführend sein.

Die Wirkung von Zimt auf die glykämische Kontrolle wurde bisher in unterschiedlicher Dosierung an kleinen Studienpopulationen mit unterschiedlicher Dauer (40 Tage-4 Monate) untersucht. Die Ergebnisse 
sind entsprechend inhomogen. Die Evidenz für eine Empfehlung der Supplementierung mit Zimt reicht nicht aus [49].

Die im Tierversuch gefundenen toxischen Effekte auf die Nierenfunktion werden kontrovers diskutiert [49]. Cassia-Zimt enthält darüber hinaus Cumarin, weshalb sich eine längerdauernde Einnahme auf die Blutgerinnung auswirken kann. Ceylon-Zimt hingegen enthält geringere Mengen Cumarin [49].

Der im Hafer enthaltene lösliche Ballastsoff $\beta$-Glucan zeigt in Studien positive Auswirkungen auf das Glukose- und Lipidprofil bei Patienten mit T2DM. Die Aufnahme von $3 \mathrm{~g} / \mathrm{d} \beta$-Glucan, enthalten in rund $60 \mathrm{~g}$ Hafer, kann bei positivem Wirkungsprofil für Typ 2 Diabetiker empfohlen werden. Für Typ 1 Diabetiker fehlen noch entsprechende Daten [50].

Funding Open access funding provided by Medical University of Vienna.

Interessenkonflikt H. Toplak hat Vorträge für die Firma Insumed absolviert und Forschungsunterstützung durch die Firma Almased erhalten. K. Schindler, J. Brix, S. Dämon, F. Hoppichler, A. Kautzky-Willer, R. Kruschitz und B. Ludvik geben an, dass kein Interessenkonflikt besteht.

Open Access Dieser Artikel wird unter der Creative Commons Namensnennung 4.0 International Lizenz (http:// creativecommons.org/licenses/by/4.0/deed.de) veröffentlicht, welche die Nutzung, Vervielfältigung, Bearbeitung, Verbreitung und Wiedergabe in jeglichem Medium und Format erlaubt, sofern Sie den/die ursprünglichen Autor(en) und die Quelle ordnungsgemäß nennen, einen Link zur Creative Commons Lizenz beifügen und angeben, ob Änderungen vorgenommen wurden.

Hinweis des Verlags Der Verlag bleibt in Hinblick auf geografische Zuordnungen und Gebietsbezeichnungen in veröffentlichten Karten und Institutsadressen neutral.

\section{Literatur}

1. Lean MEJ, Leslie WS, Barnes AC, Brosnahan N, Thom G, McCombie L, Peters C, Zhyzhneuskaya S, Al-MrabehA, Hollingsworth KG, et al. Primary care-led weight management for remission of type 2 diabetes (DiRECT): An open-label, cluster-randomised trial. Lancet. 2018;391(10120):541-51. https://doi.org/10.1016/S0140-6736(17)33102-1.

2. EASD. Evidence-based nutritional approaches to the treatment and prevention of diabetes mellitus. Nutr Metab Cardiovasc Dis. 2004;14:373-94.

3. American Diabetes Association. 5. Lifestyle management: Standards of medical carein diabetes-2019. Diabetes Care. 2019;42(Supplement 1):S46-S60. https://doi.org/10.2337/ dc19-S005.

4. Davis RM, Hitch AD, Salaam MM, Herman WH, ZimmerGaller IE, Mayer-Davis EJ. TeleHealth improves diabetes self-management in an underserved community: Diabetes TeleCare. Diabetes Care. 2010;33(8):1712-7. https://doi. org/10.2337/dc09-1919.

5. Schwingshackl L, Missbach B, Konig J, Hoffmann G. Adherence to a Mediterranean diet and risk of diabetes: A systematic review and meta-analysis. Public Health Nutr. 2015;18(7):1292-9.

6. Martinez-GonzalezMA,ZazpeI, RazquinC,Sanchez-Tainta A, Corella D, Salas-Salvado J, Toledo E, Ros E, Munoz MA,
Recondo J, et al. Empirically-derived food patterns and the risk of total mortality and cardiovascular events in the PREDIMED study. Clin Nutr. 2015;34(5):859-67.

7. Sleiman D, Al-Badri MR, Azar ST. Effect of mediterranean diet in diabetes control and cardiovascular risk modification: A systematic review. Front Public Health. 2015;3:69. https://doi.org/10.3389/fpubh.2015.00069.

8. Esposito K, Maiorino MI, Bellastella G, Chiodini P, Panagiotakos D, Giugliano D. A journey into a Mediterranean diet and type 2 diabetes: A systematic review with metaanalyses. BMJ Open. 2015;5(8):e8222. https://doi.org/10. 1136/bmjopen-2015-008222.

9. Schulze MB, Martínez-González MA, Fung TT, Lichtenstein AH, Forouhi NG. Food based dietary patterns and chronic disease prevention. BMJ. 2018 https://doi.org/10.1136/ bmj.k2396.

10. Saslow LR, Summers C, Aikens JE, Unwin DJ. Outcomes of a digitally delivered low-carbohydrate type 2 diabetes self-management program: 1-year results of a single-arm longitudinal study. Jmir Diabetes. 2018;3(3):e12. https:// doi.org/10.2196/diabetes.9333.

11. Garg A. High-monounsaturated-fat diets for patients with diabetes mellitus:Ameta-analysis. AmJClin Nutr. 1998;67(3 Suppl):577S-82S.

12. Reynolds A, Mann J, Cummings J, Winter N, Mete E, Te Morenga L. Carbohydrate quality and human health: A series of systematic reviews and meta-analyses. Lancet. 2019; https://doi.org/10.1016/s0140-6736(18)31809-9.

13. Ludwig DS, Hu FB, Tappy L, Brand-Miller J. Dietary carbohydrates: Role of quality and quantity in chronic disease. BMJ.2018;361:k2340.https://doi.org/10.1136/bmj.k2340.

14. Atkinson FS, Foster-Powell K, Brand-Miller JC. International tables of glycemic index and glycemic load values: 2008. Diabetes Care. 2008;31(12):2281-3. https://doi.org/ $10.2337 / \mathrm{dc} 08-1239$.

15. Brouns F. Overweight and diabetes prevention: Is a lowcarbohydrate-high-fat diet recommendable? Eur J Nutr. 2018;57(4):1301-12. https://doi.org/10.1007/s00394-0181636-y.

16. van WykHJ, Davis RE, Davies JS. A critical review of low-carbohydrate diets in people with Type 2 diabetes. Diabet Med. 2016;33(2):148-57.https://doi.org/10.1111/dme.12964.

17. VanItallie TB, Nufert TH. Ketones: Metabolism's ugly duckling. Nutr Rev. 2003;61(10):327-41. https://doi.org/10. 1301/nr.2003.oct.327-341.

18. Snorgaard O, Poulsen GM, Andersen HK, Astrup A. Systematic review and meta-analysis of dietary carbohydrate restriction in patients with type 2 diabetes. BMJ Open Diabetes Res Care. 2017;5(1) https:// doi.org/10.1136/bmjdrc2016-000354.

19. Dyson P. Low carbohydrate diets and type 2 diabetes: What is the latest evidence? Diabetes Ther. 2015;6(4):411-24. https://doi.org/10.1007/s13300-015-0136-9.

20. Parks EJ, Skokan LE, Timlin MT, Dingfelder CS. Dietary sugars stimulate fatty acid synthesis in adults. J Nutr. 2008;138(6):1039-46. https://doi.org/10.1093/jn/138.6. 1039.

21. Wiebe N, Padwal R, Field C, Marks S, Jacobs R, Tonelli M. A systematic review on the effect of sweeteners on glycemic response and clinically relevant outcomes. BMC Med. 2011;9:123.

22. Suez J, Korem T, Zeevi D, Zilberman-Schapira G, Thaiss CA, Maza O, Israeli D, Zmora N, Gilad S, Weinberger A, et al. Artificial sweeteners induce glucose intolerance by altering the gut microbiota. Nature. 2014;514(7521):181-6.

23. Forouhi NG, Krauss RM, Taubes G, Willett W. Dietary fat and cardiometabolic health: Evidence, controversies, and 
consensus for guidance. BMJ. 2018;361 https://doi.org/10. 1136/bmj.k2139.

24. Wheeler ML, Dunbar SA, Jaacks LM, Karmally W, MayerDavis EJ, Wylie-Rosett J, Yancy WS Jr.. Macronutrients, food groups, and eating patterns in the management of diabetes: A systematic review of the literature, 2010. Diabetes Care. 2012;35(2):434-45. https://doi.org/10.2337/dc11-2216.

25. Group ASC, Bowman L, Mafham M, Wallendszus K, Stevens W, Buck G, Barton J, Murphy K, Aung T, Haynes R, et al. Effects of n-3 fatty acid supplements in diabetes mellitus. N Engl J Med. 2018;379(16):1540-50. https://doi.org/10. 1056/NEJMoa1804989.

26. Oomen CM, Ocke MC, Feskens EJ, van Erp-Baart MA, Kok FJ, Kromhout D. Association between trans fatty acid intake and 10-year risk of coronary heart disease in the Zutphen Elderly Study: A prospective population-based study. Lancet. 2001;357(9258):746-51.

27. Mensink RP, Zock PL, Kester ADM, Katan MB. Effects of dietary fatty acids and carbohydrates on the ratio of serum total to HDL cholesterol and on serum lipids and apolipoproteins: A meta-analysis of 60 controlled trials. Am J Clin Nutr. 2003;77(5):1146-55.

28. Gardner CD, Coulston A, Chatterjee L, Rigby A, Spiller G, Farquhar JW. The effect of a plant-based diet on plasma lipids in hypercholesterolemic adults: A randomized trial. Ann Intern Med. 2005;142(9):725-33.

29. Schaefer EJ. Effects of dietary fatty acids on lipoproteins and cardiovascular disease risk: Summary. Am J Clin Nutr. 1997;65(5 Suppl):1655S-6S.

30. Krebs JD, Elley CR, Parry-Strong A, Lunt H, Drury PL, Bell DA, Robinson E, Moyes SA, Mann JI. The Diabetes Excess Weight Loss (DEWL) Trial: A randomised controlled trial of high-protein versus high-carbohydrate diets over 2 years in type 2 diabetes. Diabetologia. 2012;55(4):905-14. https:// doi.org/10.1007/s00125-012-2461-0.

31. Larsen RN, Mann NJ, Maclean E, Shaw JE. The effect of high-protein, low-carbohydrate diets in the treatment of type 2 diabetes: A 12 month randomised controlled trial. Diabetologia. 2011;54(4):731-40.

32. Gannon MC, Nuttall JA, Damberg G, Gupta V, Nuttall FQ. Effect of protein ingestion on the glucose appearance rate in people with type 2 diabetes. J Clin Endocrinol Metab. 2001;86(3):1040-7.

33. Samaha FF, Iqbal N, Seshadri P, Chicano KL, Daily DA, McGrory J, Williams T, Williams M, Gracely EJ, Stern L. A low-carbohydrate as compared with a low-fat diet in severe obesity. N Engl J Med. 2003;348(21):2074-81.

34. Belch J, MacCuish A, Campbell I, Cobbe S, Taylor R, Prescott R, Lee R, Bancroft J, MacEwan S, Shepherd J, et al. The prevention of progression of arterial disease and diabetes (POPADAD) trial: Factorial randomised placebo controlled trial of aspirin and antioxidants in patients with diabetes and asymptomatic peripheral arterial disease. BMJ. 2008;337:a1840.https://doi.org/10.1136/bmj.a1840.

35. Hasanain B, Mooradian AD. Antioxidant vitamins and their influence in diabetes mellitus. Curr Diab Rep. 2002;2(5):448-56.

36. Ellinger S, Stehle P. Efficacy of vitamin supplementation in situations with wound healing disorders: Results from clinical intervention studies. Curr Opin Clin Nutr Metab Care. 2009;12(6):588-95. https://doi.org/10.1097/ MCO.0b013e328331a5b5.
37. Wilkinson EA. Oral zinc for arterial and venous leg ulcers. Cochrane Database Syst Rev. 2014;9:CD1273. https://doi. org/10.1002/14651858.CD001273.pub3.

38. Kleefstra N, Houweling ST, Jansman FGA, Groenier KH, Gans ROB, Meyboom-de Jong B, Bakker SJL, Bilo HJG. Chromium treatment has no effect in patients with poorly controlled, insulin-treated type 2 diabetes in an obese western population:A randomized, double-blind, placebocontrolled trial. Diabetes Care. 2006;29(3):521-5.

39. Rayman MP. Selenium and human health. Lancet. 2012;379(9822):1256-68. https://doi.org/10.1016/S01406736(11)61452-9.

40. Korpelainen R, Korpelainen J, Heikkinen J, Vaananen K, Keinanen-Kiukaanniemi S. Lifelong risk factors for osteoporosis and fractures in elderly women with low body mass index-A population-based study. Bone. 2006;39(2):385-91. https://doi.org/10.1016/j.bone.2006.01.143.

41. Guerrero-Romero F, Rodríguez-Morán M. Hypomagnesemia, oxidative stress, inflammation, and metabolic syndrome. Diabetes Metab Res Rev. 2006;22(6):471-6. https:// doi.org/10.1002/dmrr.644.

42. Guerrero-Romero F, Rodriguez-Moran M. Low serum magnesium levels and metabolic syndrome. Acta Diabetol. 2002;39(4):209-13. https://doi.org/10.1007/s005920200 036.

43. Peters KE, Chubb SA, Davis WA, Davis TM. The relationship between hypomagnesemia, metformin therapy and cardiovascular disease complicating type 2 diabetes: The Fremantle Diabetes Study. PLoS ONE. 2013;8(9):e74355. https://doi.org/10.1371/journal.pone.0074355.

44. Haglin L, Tornkvist B, Backman L. Prediction of allcause mortality in a patient population with hypertension and type $2 \mathrm{DM}$ by using traditional risk factors and serum-phosphate,-calcium and-magnesium. Acta Diabetol. 2007;44(3):138-43. https://doi.org/10.1007/s00592-0070254-6.

45. Hata A, Doi Y, Ninomiya T, Mukai N, Hirakawa Y, Hata J, Ozawa M, Uchida K, Shirota T, Kitazono T, et al. Magnesium intake decreases Type 2 diabetes risk through the improvement of insulin resistance and inflammation: The HisayamaStudy. Diabet Med.2013;30(12):1487-94.https:// doi.org/10.1111/dme.12250.

46. Dong JY, Xun P, He K, Qin LQ. Magnesium intake and risk of type 2 diabetes: Meta-analysis of prospective cohort studies. Diabetes Care. 2011;34(9):2116-22. https://doi.org/ 10.2337/dc11-0518.

47. Deutsche Gesellschaft für Ernährung, Österreichische Gesellschaft für Ernährung, Schweizerische Gesellschaft für Ernährung.D-A-CHReferenzwertefür dieNährstoffzufuhr. 1. Aufl. Frankfurt/Main: Umschau Verlag; 2012. 4. korrigierter Nachdruck.

48. Manya K, Champion B, Dunning T. The use of complementary and alternative medicine among people living with diabetes in Sydney. Bmc Complement Altern Med. 2012;12:2.https://doi.org/10.1186/1472-6882-12-2.

49. Medagama $A B$, Bandara R. The use of complementary and alternative medicines (CAMs) in the treatment of diabetes mellitus: Is continueduse safe and effective? Nutr J. 2014;13:102.https://doi.org/10.1186/1475-2891-13-102.

50. Shen XL, Zhao T, Zhou Y, Shi X, Zou Y, Zhao G. Effect of oat beta-Glucan intake on Glycaemic control and insulin sensitivity of diabetic patients: A Meta-analysis of randomized controlled trials. Nutrients. 2016;8(1) https:// doi.org/ $10.3390 /$ nu8010039. 